\title{
Spontaneously Recanalised Coronary Thrombus in the Left Anterior Descending Artery Presenting as Ventricular Tachycardia
}

\author{
Jie Man Low, ${ }^{1}$ Noah Kimit, ${ }^{1}$ Rizwan Rashid² and Magdi El-Omar, ${ }^{1,2}$ \\ 1. School of Medical Sciences, University of Manchester, Manchester, UK; 2. Department of Cardiology, Manchester Heart Centre, \\ Manchester Royal Infirmary, Manchester University NHS Foundation Trust, Manchester, UK
}

DOI: https://doi.org/10.17925/HI.2020.14.2.123

S pontaneously recanalized coronary thrombus (SRCT), also known as honeycomb, lotus root or Swiss-cheese lesion, is an increasingly recognised finding in patients undergoing coronary angiography. It is thought to arise from partial resorption of an initially occlusive thrombus. Most patients present with angina or exertional breathlessness. We describe a case of a 69-year-old patient who presented with ventricular tachycardia and was found to have SRCT in the left anterior descending artery on coronary angiography. Echocardiography and left ventricular (LV) angiography showed an akinetic, aneurysmal, thin-walled LV apex, diagnostic of an old anterior infarct. We highlight the role of optical coherence tomography in making the diagnosis and discuss the available management options of this condition.

\section{Keywords}

Spontaneously recanalized coronary thrombus, honeycomb lesion, lotus root lesion, ventricular tachycardia, optical coherence tomography

Disclosure: Jie Man Low, Noah Kimit, Rizwan Rashid and Magdi El-Omar have no financial or non-financial relationships or activities to declare in relation to this article.

Review Process: Double-blind peer review.

Compliance with Ethics: Informed consent was obtained from the patient described in this case study.

Authorship: The named authors meet the International Committee of Medical Journal Editors (ICMJE) criteria

for authorship of this manuscript, take responsibility for the integrity of the work as a whole, and have given final approval for the version to be published.

Access: This article is freely accessible at touchCARDIO.com (c) Touch Medical Media 2020

Received: 11 June 2020

Accepted: 14 October 2020

Published Online: 22 December 2020

Citation: Heart International. 2020;14(2):123-8

Corresponding Author: Dr Magdi El-Omar,

Manchester Heart Centre, Manchester Royal Infirmary,

Manchester University NHS Foundation Trust,

Manchester, UK. E: magdi.el-omar@mft.nhs.uk

Support: No funding was received in

the publication of this article.
Spontaneously recanalised coronary thrombus (SRCT), informally known as Swiss-cheese, honeycomb, lotus root or spiderweb lesion, is an increasingly recognised pathology in patients undergoing coronary angiography. Most patients with SRCT present with angina or angina equivalent. ' We describe a case of SRCT in a patient who presented with ventricular tachycardia (VT) without a previous history of ischaemic heart disease. We discuss the aetiology and management of this condition and highlight the role of optical coherence tomography (OCT) in making the diagnosis.

\section{Case study}

A 69-year-old lady presented to our emergency department with central crushing chest pain and palpitations. An ECG showed VT at a rate of 170 bpm (Figure 1). She gave a past medical history of paroxysmal atrial fibrillation, for which she was on warfarin, and a cerebrovascular accident 3 years ago, which left her with a fixed flexion deformity in the right arm and expressive dysphasia. She denied any past history of myocardial infarction or chest pain. She consumed 20 units of alcohol per week and smoked 20 cigarettes per day. Her medications on admission were warfarin and citalopram. Blood tests were unremarkable, apart from a raised high sensitivity Troponin- $T$ at $594 \mathrm{ng} / \mathrm{L}$.

Despite the VT, the patient was haemodynamically stable. She was treated with intravenous (IV) amiodarone (300 mg bolus) and an IV infusion of magnesium sulfate, which successfully cardioverted her to sinus rhythm within 2 hours. A repeat ECG in sinus rhythm revealed $\mathrm{T}$-wave inversion in the anterolateral and inferior leads, as well as loss of $\mathrm{R}$ waves in $\mathrm{V} 1-\mathrm{V} 3$, indicative of an old anteroseptal myocardial infarction (Figure 2). Echocardiography revealed a thin-walled, akinetic, aneurysmal apex with overall moderate left ventricular (LV) systolic dysfunction (estimated LV ejection fraction 40\%) (Figure 3). No intra-cardiac thrombus was identified. Her warfarin was discontinued and she was started on aspirin, clopidogrel, fondaparinux, bisoprolol and atorvastatin.

Four days later, she underwent cardiac catheterisation via a left radial approach. LV angiography revealed an akinetic/dyskinetic, aneurysmal apex, severe hypokinesia of the anterior wall and good basal contraction (Figure 4). Selective coronary angiography (Figure 5A-D) revealed mild atheroma in the right coronary artery and no significant disease in the left main stem and left circumflex. The proximal third of the left anterior descending artery (LAD) was mildly diseased. In the middle third of this vessel, there was a long hazy segment with an irregular lumen outline and, in certain projections, a line transecting it along its longitudinal axis, consistent with a chronic healed dissection (Figure $5 B-D$ ). The distal third of the vessel was healthy with thrombolysis in myocardial infarction flow grade III. 
Figure 1: Electrocardiogram on admission showing ventricular tachycardia at a rate of $170 \mathrm{bpm}$

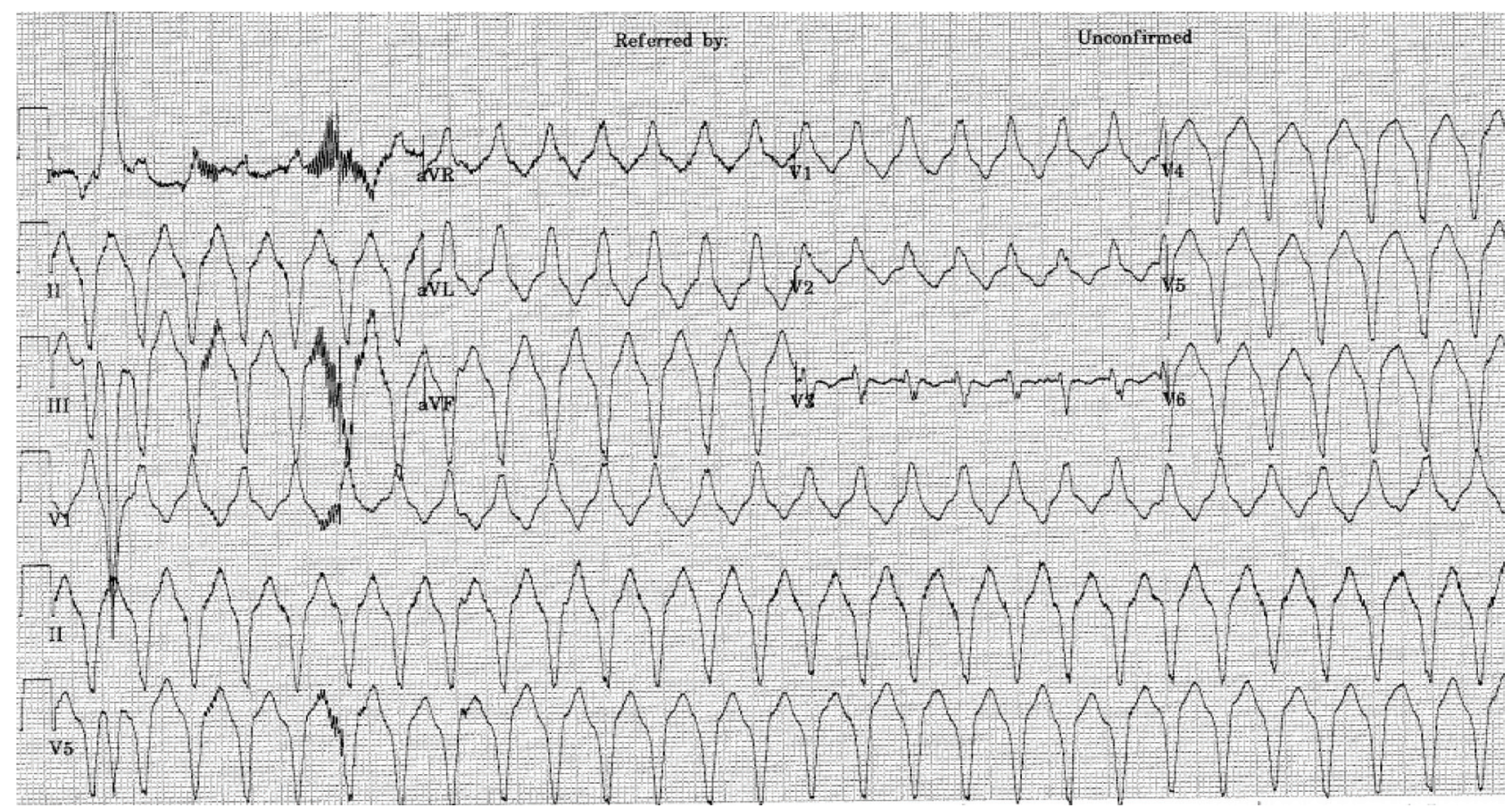

Figure 2: Electrocardiogram following pharmacological cardioversion showing sinus rhythm, loss of R waves in V1-V3 and T-wave inversion in the anterolateral and inferior leads

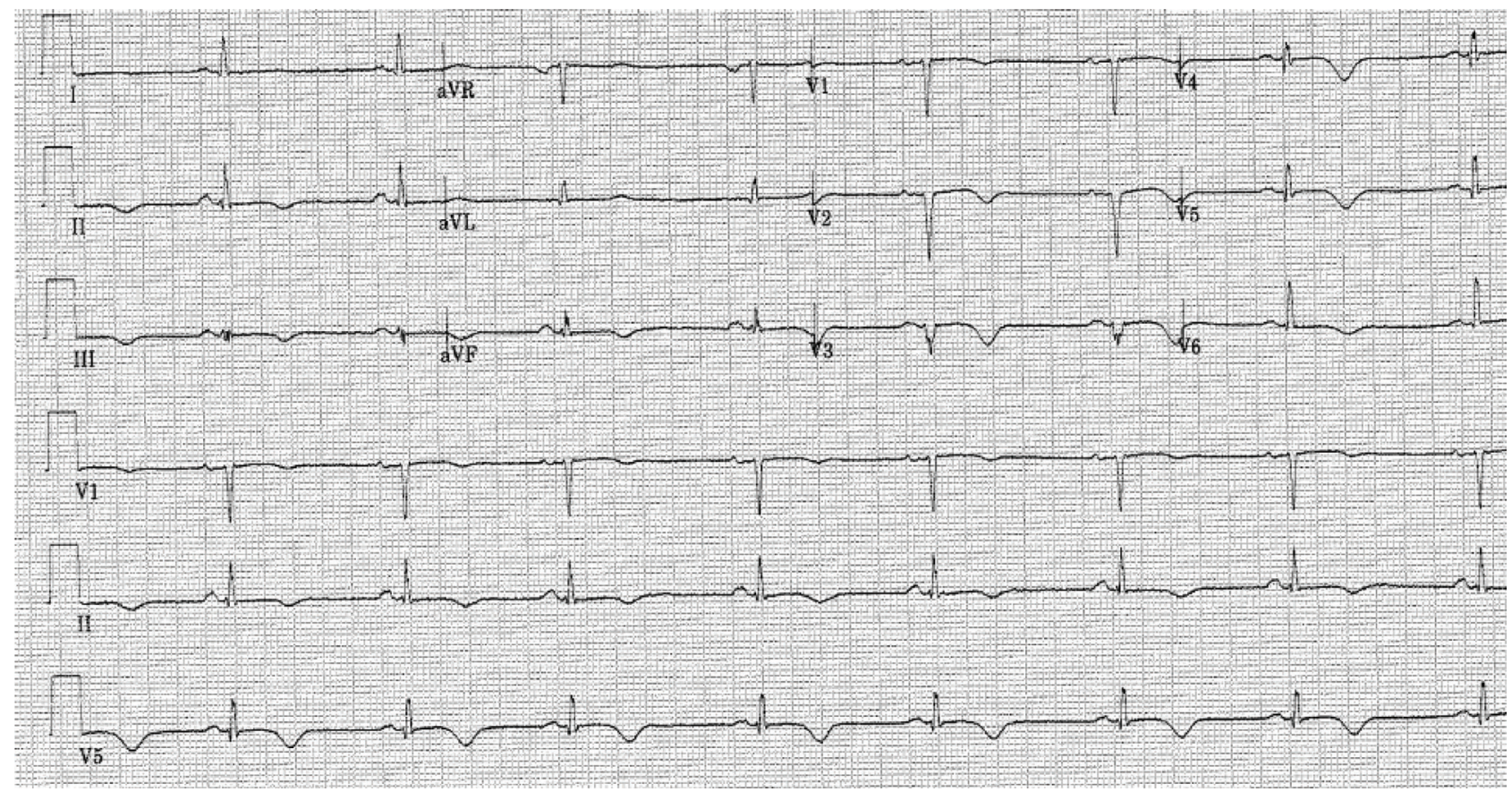

In order to shed further light on this lesion, we proceeded to intravascular imaging with OCT. An IV bolus of heparin (100 units $/ \mathrm{kg}$ ) was administered and the left main stem was cannulated using a 6F JL3.5 guide catheter. A 0.014 Sion Blue guidewire (Asahi Intecc USA, Inc., Santa Ana, CA, USA) could not be advanced past the lesion, but this was successfully achieved using a 0.014 Whisper ${ }^{\circledR}$ MS guidewire (Abbott Vascular, Abbott Park, IL, USA). OCT pullback was performed using a Dragonfly ${ }^{\top M}$ OPTIS imaging catheter (St. Jude Medical, St. Paul, MN, USA). This demonstrated a honeycomb-like appearance in the mid-LAD, comprised of multiple cavities of various sizes and shapes, separated by thin septa of high 
Figure 3: Apical 4 chamber (A) and apical 2 chamber (B) echocardiographic views showing a thin-walled, aneurysmal left ventricular apex (arrows)

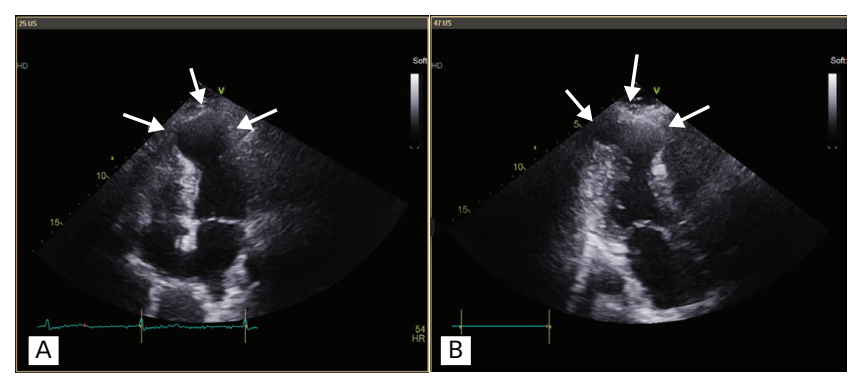

signal intensity and low signal attenuation (Figure 6A-F). This appearance is pathognomonic of SRCT.

The lesion length was approximately $30 \mathrm{~mm}$ as can be appreciated on the longitudinal mode reconstruction (Figure 7). There was no evidence of fresh thrombus or disruption to the intimal layer to suggest coronary dissection or ruptured plaque. Although stenting of the lesion would have been technically feasible, we decided against this given the absence of symptoms of chest pain prior to admission, and the likely non-viable nature of the infarcted territory, as suggested by the echocardiography. A cardioverter-defibrillator was implanted 4 days later and she was discharged home the following day on lifelong warfarin, clopidogrel, bisoprolol and atorvastatin. She remains well and completely asymptomatic 2 years following discharge.

\section{Discussion}

A hazy lesion is commonly encountered on coronary angiography, and may be due to fresh thrombus, calcium, spontaneous coronary dissection, ulcerated plaque or a combination of these. Another increasingly recognised cause is a partially recanalised, organised coronary thrombus, as exemplified by the present case. When an occlusive thrombus forms within a vessel (perhaps as a result of plaque rupture or spontaneous dissection), it may remain occlusive long-term (i.e. forms a chronic total occlusion), or may undergo partial resorption over weeks to months, forming a highly complex structure of inter-communicating pockets and channels, separated by thin fibrotic septa. These partially recanalized thrombi are a common finding in autopsy studies of patients who die of coronary thrombosis, with one series reporting them in up to $40 \%$ of cases. $^{2}$

To our knowledge, this is the first case report of SRCT presenting as VT. We believe the chest pain that triggered the patient's admission to hospital was secondary to the VT, which in turn was related to scar tissue from an old anterior infarct. This is suggested by the echocardiographic finding of an akinetic, aneurysmal and thin-walled LV apex. The initially occlusive LAD thrombus is likely to have recanalised partially and gradually over time, culminating in the formation of SRCT. We opted to manage the lesion conservatively, given lack of anginal symptoms prior to admission and the unlikely presence of viable myocardium, or indeed reversible ischaemia, within the infarcted territory.

When visualised by OCT, SRCT lesions have been variably described as honeycomb, lotus root, Swiss-cheese or spiderweb in appearance because of obvious resemblance to these structures (Figure $8 \mathrm{~A}-\mathrm{C}$ ). The number, shape and size of channels, cavities and septa within these lesions are highly variable, and this is reflected in a wide range
Figure 4: Left ventricular angiography (end-systolic frame) showing an aneurysmal apex (arrows) with good basal contraction

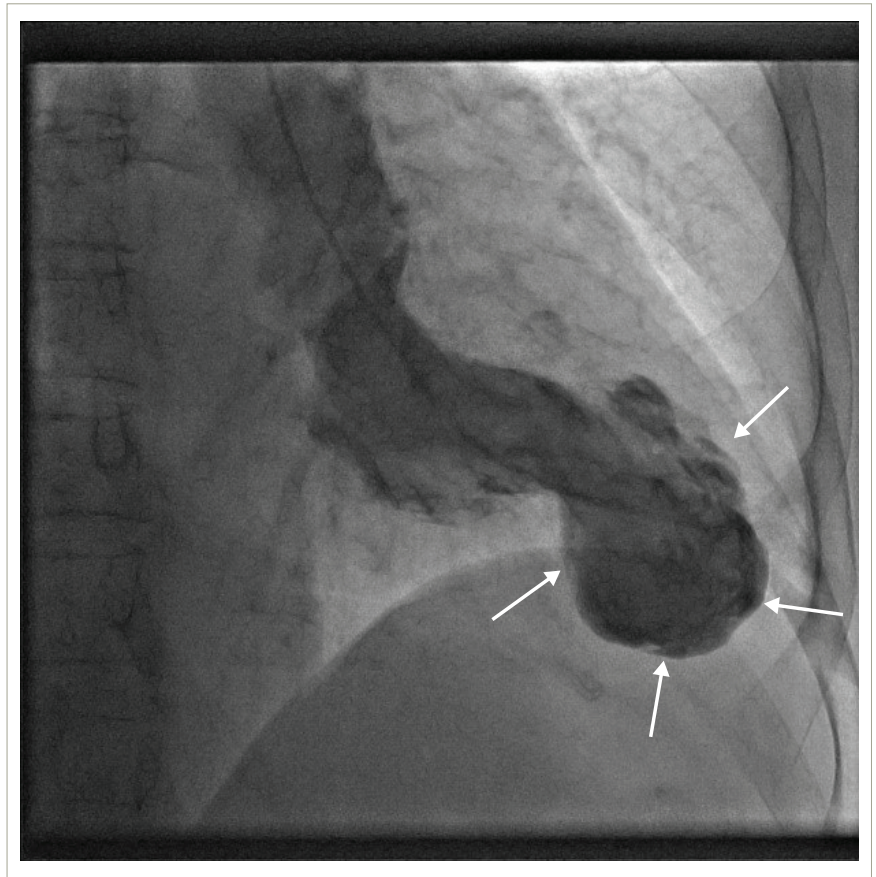

of stenosis severity, as seen on coronary angiography and/or OCT. ${ }^{3}$ Angiographically, SRCT is most commonly mistaken for a chronic healed dissection because of the longitudinal lines that transect it as dye travels through the microchannels. In the largest reported case series of SRCT confirmed on OCT, Souteyrand et al. described three distinct angiographic appearances: braided, pseudo-dissection and hazy, with the braided type (multiple linear and/or interlaced structures) being most common. ${ }^{3}$ However, the clinical utility of this classification is unclear given equivalent clinical outcomes for all three types following percutaneous coronary intervention $(\mathrm{PCl}){ }^{3}$

SRCT has been described in all major epicardial coronary arteries, including, very occasionally, in more than one artery in the same patient. ${ }^{4}$ The majority are thought to arise from in situ thrombosis, but recanalization of an embolic thrombus has also been described..$^{5}$ An embolic origin is quite plausible in the present case, given a past history of paroxysmal atrial fibrillation and cerebrovascular accident, and absence of significant coronary artery disease on angiography.

The need for PCI to SRCT should be guided not only by the angiographic severity of the lesion, but also the presence of symptoms and/or evidence of reversible myocardial ischaemia. Reliance on angiographic severity alone to judge the need for $\mathrm{PCl}$ is misleading, given poor correlation with symptomatic status. Souteyrand et al. found that the stenosis severity of SRCT on quantitative coronary analysis varied between $11 \%$ and $100 \%$, yet all patients were symptomatic with angina/dyspnoea and/or had evidence of reversible myocardial ischaemia on functional testing. ${ }^{3}$

Given their highly complex morphology, one might anticipate technical difficulty in negotiating SRCT lesions with guidewires, balloons, stents or intravascular imaging devices. In the present case, there was some difficulty wiring the lesion, but the subsequent advancement of the OCT catheter was accomplished easily. Similar technical difficulties have been reported by some, ${ }^{6}$ but not all, operators. ${ }^{3}$ 
Figure 5: Selective coronary angiography of the right $(A)$ and left $(B-D)$ coronary arteries
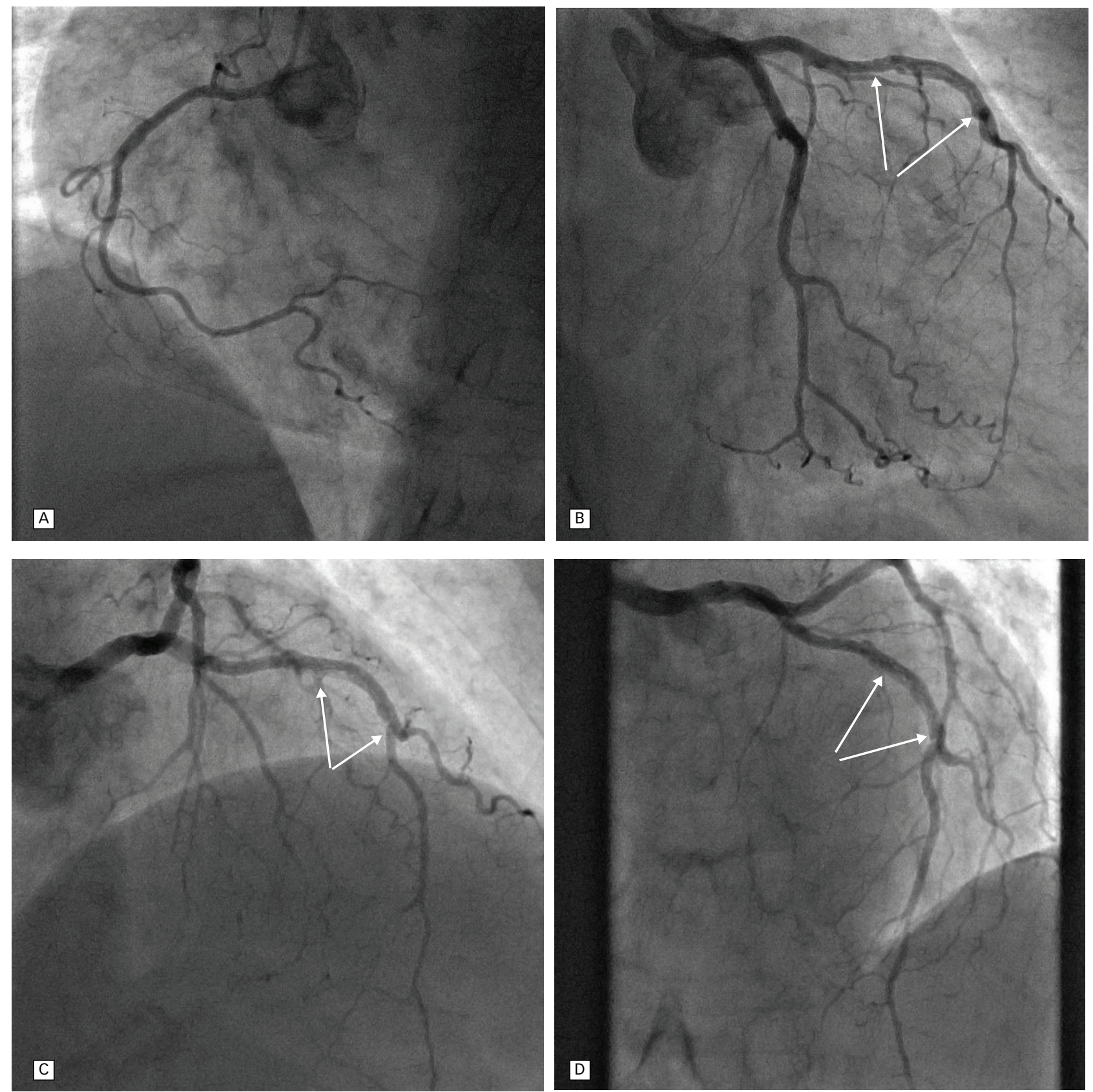

The white arrows point to a long hazy segment in the mid-left anterior descending artery which has the appearance of a chronic healed dissection; (B) anterior-posterior caudal; (C) right anterior oblique cranial; (D) anterior-posterior cranial.

The acute procedural results following stent implantation in SRCT appear favourable, with one case series reporting no malapposition, under-deployment or edge dissection on post-stenting OCT. ${ }^{3}$ Treatment of these lesions with drug-eluting stents, drug-eluting balloons and bioresorbable scaffolds has been described, with generally favourable short- and long-term outcomes.? One case series, however, reported an increased risk of side branch compromise associated with worse clinical outcomes following stenting of these lesions. ${ }^{8}$

The present case highlights the role of intravascular imaging in elucidating the nature of an angiographically hazy coronary lesion. In this regard, OCT is superior to intravascular ultrasound (IVUS), given its higher spatial resolution (10 $\mu \mathrm{m}$ versus $100 \mu \mathrm{m}$ for IVUS) and near-histological image quality. ${ }^{910}$ Furthermore, good image quality can be achieved safely with OCT, despite the OCT catheter occluding the channel it traverses in many cases. ${ }^{3}$

With full characterisation of an ambiguous coronary lesion, OCT can guide the next step in management. For instance, a hazy lesion due to thrombus may require adjunctive use of thrombus aspiration $\pm \| \mathrm{lb} / \mathrm{llla}$ receptor antagonist prior to stenting, whereas a heavily calcified lesion may require aggressive preparation with non-compliant balloon pre-dilatation and/or use of specialised devices, such as cutting balloons, scoring balloons, rotational atherectomy or intravascular lithotripsy. ${ }^{11}$ Demonstration of a spontaneous coronary artery dissection, on the other hand, suggests conservative 
Figure 6: Serial cross-sectional optical coherence tomography images of the hazy segment in the mid-left anterior descending artery
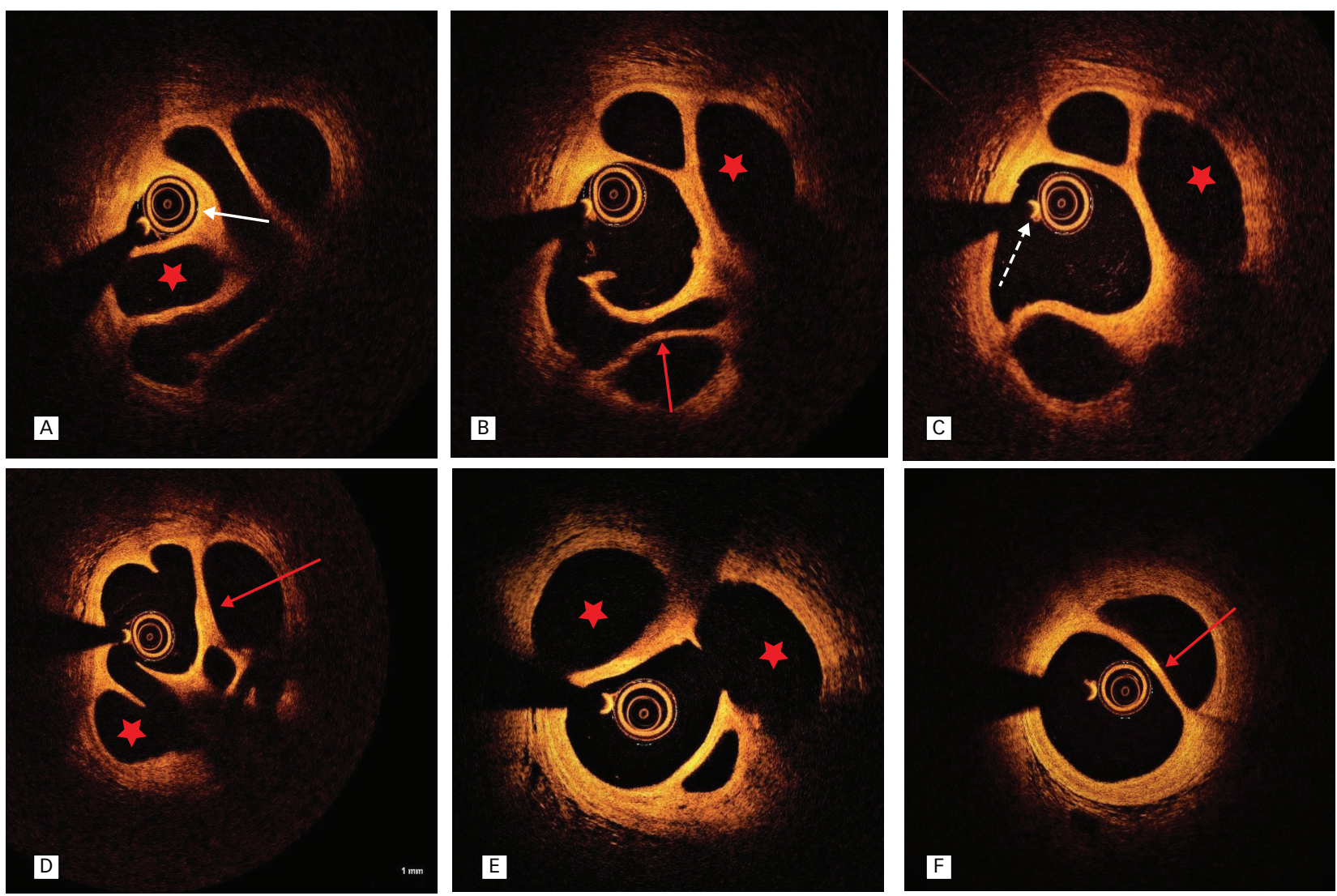

The red stars (A-E) show a complex morphology of inter-communicating cavities/micro-channels of various shapes and sizes, separated by thin septa of high signal intensity and low signal attenuation (shown by the red arrows [B, D, F]). Note 'wedging' of the OCT catheter in a micro-channel (solid arrow [A]). Note guidewire and its shadow (dotted arrow [C]).

Figure 7: Longitudinal mode optical coherence tomography image of the hazy mid-left anterior descending segment

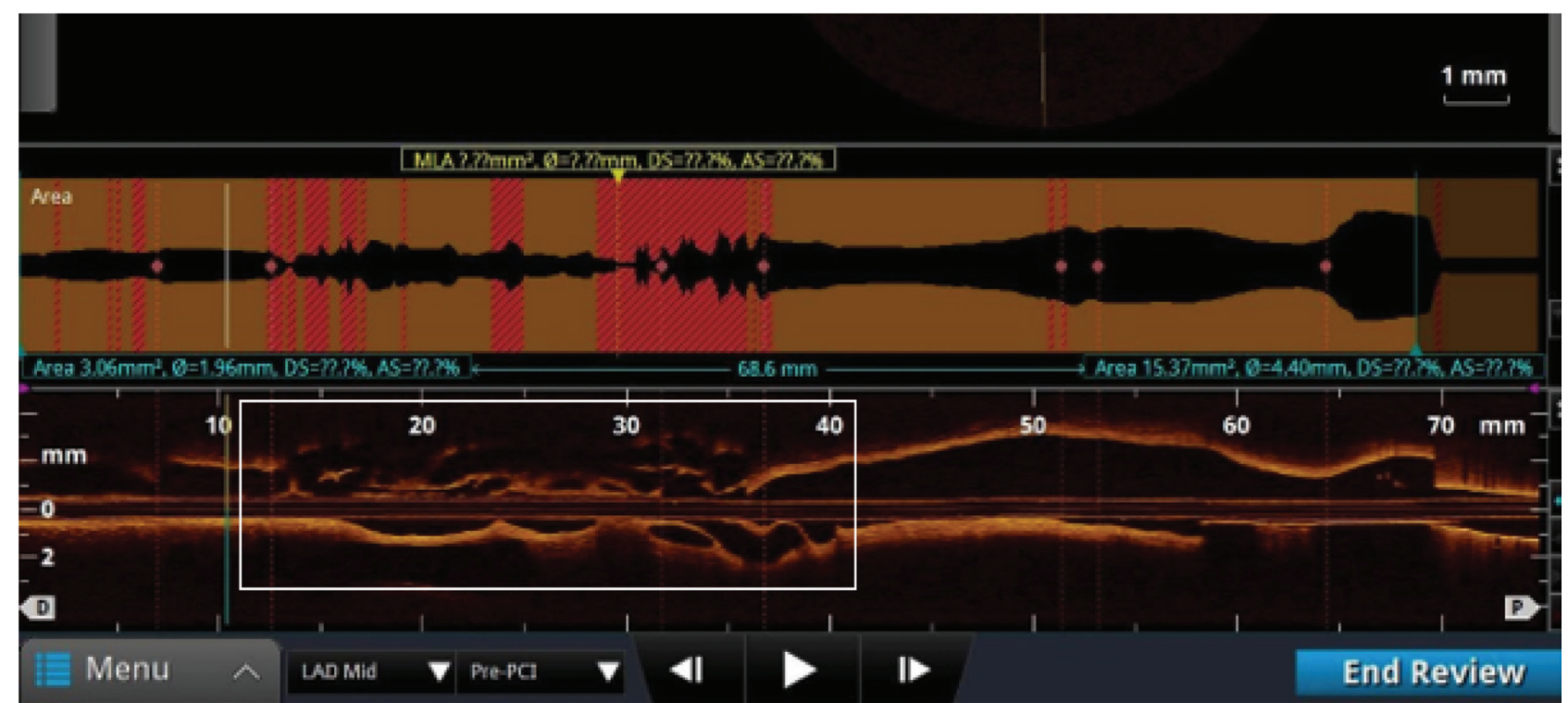

The white box shows a complex structure of inter-communicating channels within the spontaneously recanalised coronary thrombus. 
Figure 8: Structures that have a similar appearance to a spontaneously recanalised coronary thrombus as seen on optical coherence tomography
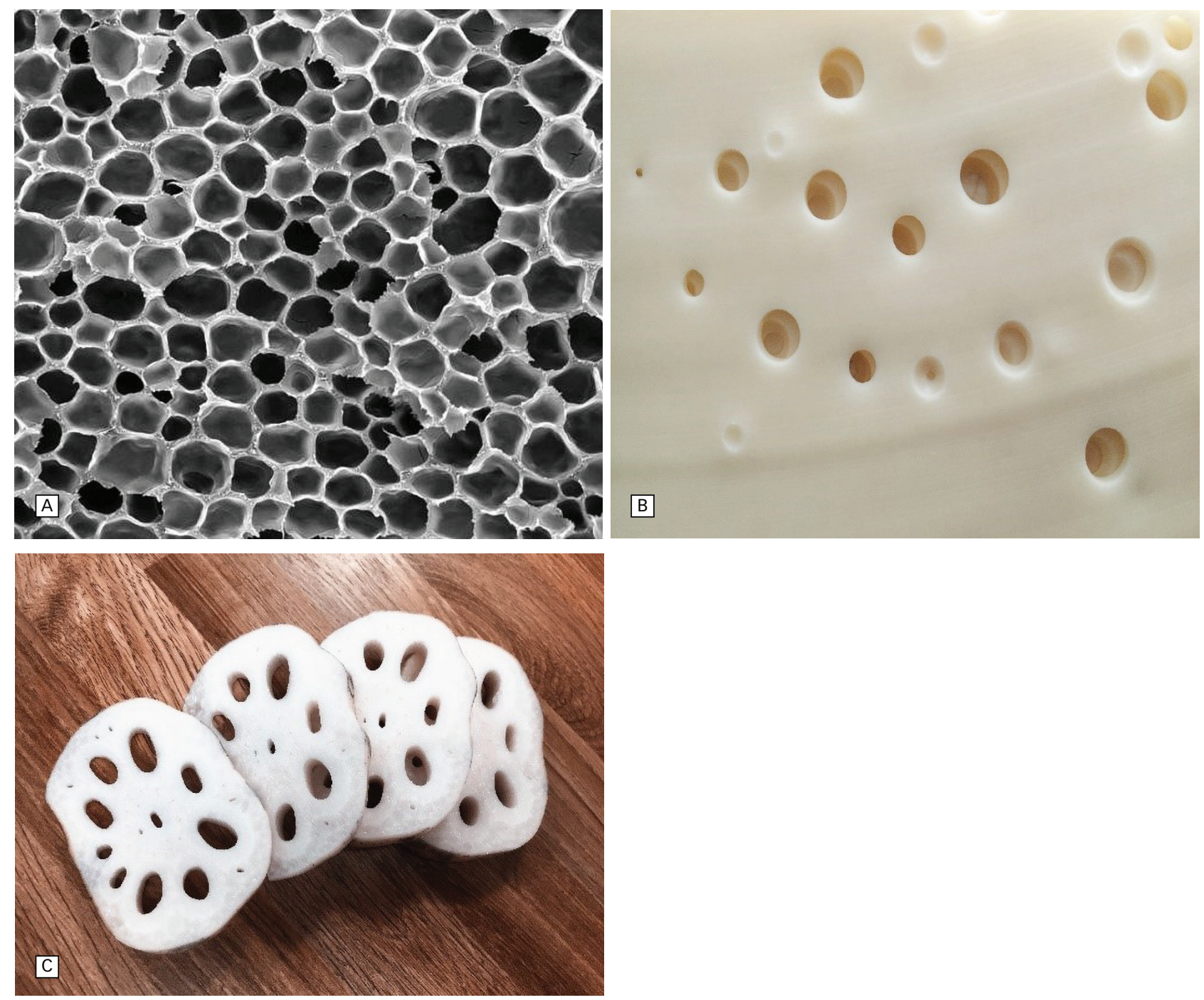

A. Honeycomb; B. Swiss cheese; C. lotus root

management as the most appropriate strategy, given that most such lesions heal spontaneously. ${ }^{12,13}$

\section{Conclusion}

SRCT is a rare, but increasingly recognised pathology that may present with angina, exertional dyspnoea or, as described in the present case, VT. The diagnosis requires intravascular imaging for confirmation as multiple other pathologies give a similar appearance on coronary angiography. Given its high resolution and near-histological image quality, OCT is the intravascular imaging modality of choice for elucidating the cause of an angiographically hazy coronary lesion. While the exact aetiology of SRCT remains poorly understood, its management depends on the presence of symptoms and/or demonstration of reversible myocardial ischaemia on stress testing. If neither is present, a conservative approach is reasonable, as shown in the present case. $]$
1. Kang SJ, Nakano M, Virmani R, et al. OCT findings in patients with recanalization of organized thrombi in coronary arteries. JACC Cardiovasc Imaging. 2012:5·725-32.

2. Friedman $\mathrm{M}$. The coronary canalized thrombus: provenance, structure, function and relationship to death due to coronary artery disease. Br J Exp Pathol. 1967:48:556-7.

3. Souteyrand G, Valladier M, Amabile N, et al. Diagnosis and management of spontaneously recanalized coronary thrombus guided by optical coherence tomography - lessons from the French "Lotus Root" Registry. Circ I. 2018:82:783-90.

4. Kadowaki H Taguchi E Kotono Y et al. A lotus root-like appearance in both the left anterior descending and right coronary arteries. Heart Vessels. 2016;31:124-8.

5. Toutouzas K, Karanasos A, Stathogiannis K, et al. A honeycomb-like structure in the left anterior descending coronary artery: Demonstration of recanalized thrombus by optical coherence tomography. JACC Cardiovasc Interv. 2012;5:688-9.

6. Niizeki T, Ikeno E Iwayama T, Watanabe M. Difficult wiring of recanalized thrombotic lesion in the right coronary artery a

7. Lin M, SuZ Z L I Nie R, Wang J. Honeycomb-like structure in the right coronary artery treated with a drug-eluting stent: a case report and literature review. I Int Med Res. 2018;46:2008-13.

8. Xu T, Shrestha R, Pan T, et al. Anatomical features and clinical outcome of a honeycomb-like structure in the coronary artery: reports from 16 consecutive patients. Coron Artery Dis. 2020;31:222-9

9. Garcia-Garcia HM, Gogas BD, Serruys PW, Bruining N. IVUS-based imaging modalities for tissue characterization: similarities and differences. Int I Cardiovasc Imaging. 2011;27:215-24.

10. Prati F, Guagliumi G, Mintz GS, et al. Expert review document part 2: methodology, terminology and clinical applications of optical coherence tomography for the assessment of interventional procedures. Eur Heart 2012:33.2513-20.

11. Ali ZA, Karimi Galougahi K, Maehara A, et al. Intracoronary optical coherence tomography 2018: current status and future directions. JACC Cardiovasc Interv. 2017:10:2473-87.

12. Alfonso F, Paulo M, Gonzalo N, et al. Diagnosis of spontaneous coronary artery dissection by optical coherence tomography. J Am Coll Cardiol. 2012;59:1073-9.

13. Garcia-Guimarães M, Bastante T, Antuña P, et al. Spontaneous coronary artery dissection: mechanisms, diagnosis and management. Eur Cardiol. 2020;15:1-8. 